\title{
HALO REDUCTION BY MEANS OF NON LINEAR OPTICAL ELEMENTS IN THE NLC FINAL FOCUS SYSTEM*
}

\author{
R. Brinkmann, DESY, P. Raimondi, A. Seryi, SLAC
}

\section{Abstract}

In the Beam Delivery Systems (BDS) for linear colliders that have been designed or built, collimators (or scrapers) are used to suppress backgrounds due to the beam halo. Off-energy and off-axis particles are stopped on the collimator jaws, physically limiting the acceptance of the system. This concept does not scale well to higher beam energy, higher intensity or lower emittance. The increased beam density requires longer and more demanding collimator regions. In contrast, this paper studies the possibility of inserting non-linear optical elements into the Final Focus in order to effectively increase the acceptance of the system. This technique could make the traditional collimation scheme obsolete so that only protection collimators would be needed.

\section{INTRODUCTION}

In a linear collider, two main requirements drive the need for collimation. All the beam particles must be contained within the physical dimensions of the beam pipe in the Final Focus (FF), particularly in the Final Doublet (FD). The synchrotron radiation emitted by the beam across the FD should not hit any element in the Interaction Region (IR), particularly the vertex detector.

There are three different methods for attacking this problem: a) suppress all of the beam particles that do not satisfy the conditions above by means of collimators; $b$ ) increase the dynamic aperture of the FF by reducing the non-linearities of the system; c) increase the dynamic aperture of the FF by adding opportunistic non-linearities into the system. Until recently, only the first method has been extensively developed. The chromatic properties of the FF completely dominate the optics design but have not been exploited for collimation.

This paper will present a minor modification of the current NLC FF design [1], which uses the latter two methods to greatly reduce the beam scraping required. With this system, the collimator region can be much shorter and simpler than the present NLC design [2]. The earlier NLC ZDR had an even longer, more complicated collimator region [3].

\section{MINIMIZATION OF NON-LINEARITIES}

The NLC FF has been extensively analyzed to explore the behavior of large amplitude offset particles. A few modifications have been made to increase the stay-clear of the system. The present FF has a larger dynamic aperture than other equivalent designs, primarily because

*Work supported by the U.S. Department of Energy, Contract DE-AC03-76SF00515 the chromaticity generated by the FD is corrected as locally as possible, by means of 2 sextupoles interleaved with the FD. The system proposed in [1] has been further enhanced to minimize higher-order aberrations. The new optics is shown in Fig.1. Figure 2 shows the beam distributions at the entrance of the FD for an incoming beam with emittances about $10^{4}$ times the nominal NLC values. Figure $2 \mathrm{c}$ shows that the linear behavior of the system is greatly improved in the modified FF.

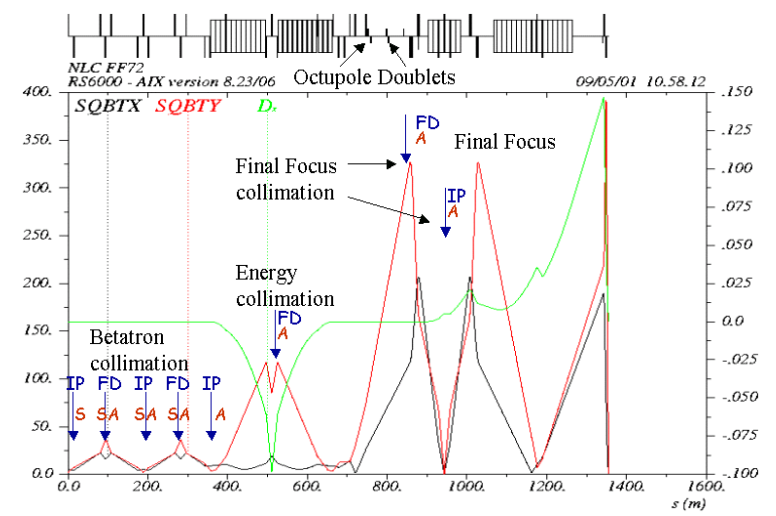

Figure 1: Proposed optics for the NLC BDS.

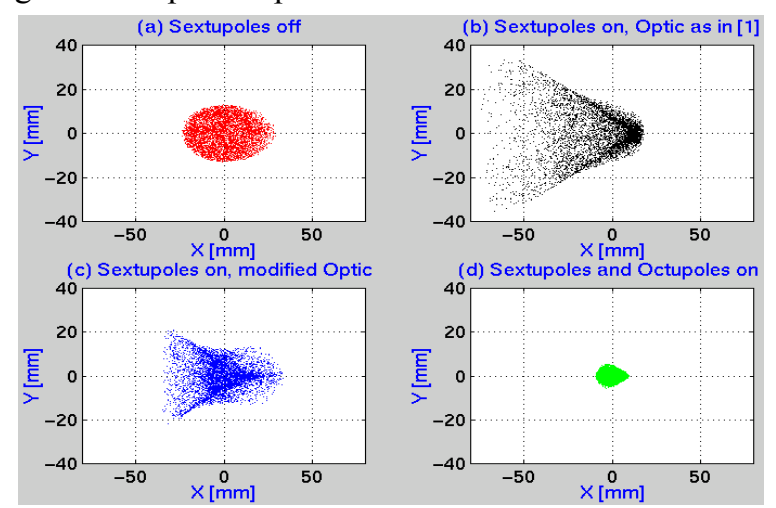

Figure .2: Beam at the entrance of the Final Doublet assuming a flat input beam distribution with maxima $\left(\sigma_{x}, \sigma_{x^{\prime}}, \sigma_{y}, \sigma_{y^{\prime}}, \mathrm{dE} / \mathrm{E}\right)=(24 \mu \mathrm{m}, 1.0 \mathrm{mrad}, 5.4 \mu \mathrm{m}, 4.4 \mathrm{mrad}, 3 \%)$. The input beam dimensions assume the IP $\beta$ 's.

\section{INSERTION OF NON-LINEARITIES}

An apparent drawback of the local chromaticity compensation in the new NLC FF design is that non-zero dispersion across the doublet is required. This would seem to imply that tighter collimation of off-energy particles would be needed. It has already been shown in [1] that this is not correct. The tightest limit on the dynamic aperture of the FF is the higher-order chromogeometric aberrations, which are very small in this design. In addition, the non-linear chromo-geometric aberrations generated by the sextupoles in this system actually result in a smaller beam size in spite of the first 
order dispersion. As shown in Fig.3b, there is also some third order dispersion across the doublet, which causes $\beta_{x}$ to decrease for off energy particles. Both effects more than cancel the increased size due to the first order dispersion. A far off-energy beam halo will not behave much worse then a monochromatic beam. The energy dependence of the beam halo vanishes almost entirely with the octupoles (Fig.3e/3f) described later.

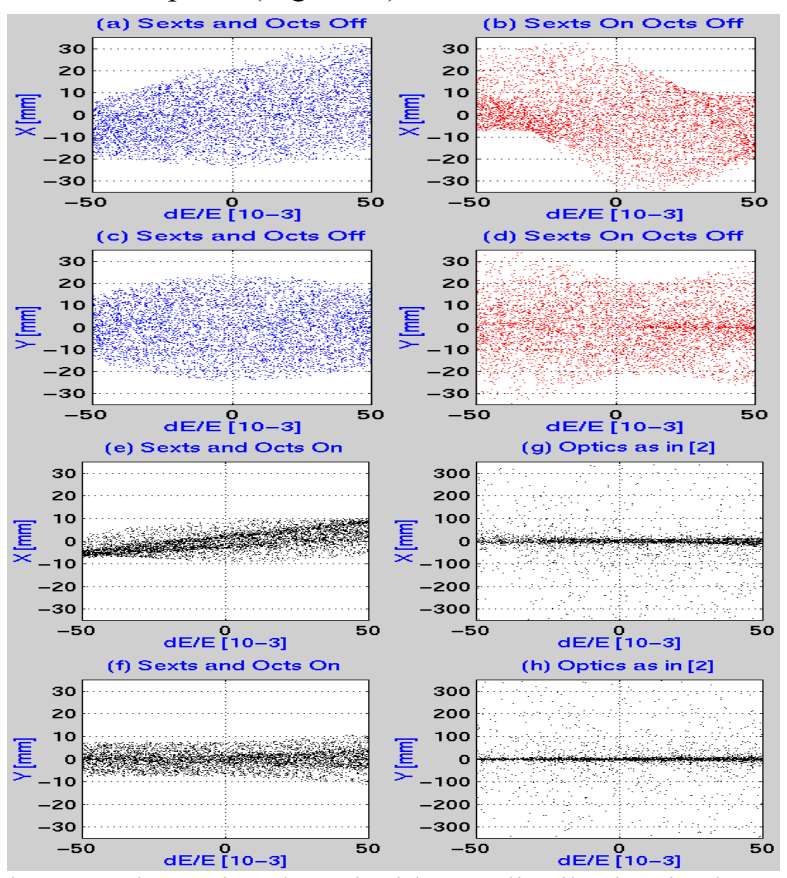

Fig.3: Horizontal and vertical beam distribution in the FD at the maximum $\beta_{x}$ and $\beta_{y}$ vs. the beam energy. Transverse phase space distributions are as in Fig.2.

The stay-clear of the BDS can be further increased by additional non-linear optical elements. In [1] it has been shown that this system has a very large aperture in both the horizontal and vertical plane for particles at the IP phase. This is a crucial point for a non-linear halo folding, since in general non-linear elements increase the effective emittance of the beam. For the technique to work, some "free" phase space must be available to be able to shift particles from a harmful region of the phase space to a safe region. Octupoles provide a possible method for nonlinear collimation. The use of octupoles for non-linear control of beam tails has long history $[4,5]$; it has been investigated for the linac [7] and for the Final Focus $[6,7,8,9]$, though the advantages of nonlinear collimation in FF become possible only recently, in a new FF that has good control of high order aberrations [1].

For the simple case of collimation only in the horizontal plane, Fig.4 shows that an octupole placed just downstream of the $\beta$ Matching Section ( $\beta M S$ ) of the FF, can fold the beam phase space distribution at the entrance of the FD. Particles that would have crossed the doublet with large offsets will now have a much smaller position offset but some angle. This angle is still too small to increase the FD synchrotron radiation swath, whose span is dominated by the focusing in the FD. The folding can be described by the equation:

$$
X_{F D}=X_{F D}^{o}+\frac{R_{12}}{R_{11}^{3}} K_{o c t} X_{F D}^{o 3}
$$

$R_{i j}$ are the first order transfer matrix elements between the octupole and the FD, $K_{o c t}$ is octupole strength, $X_{F D}^{o}$ and $X_{F D}$ are the particle offsets at the FD with the octupole on and off, respectively. For simplicity we assume that the $X^{\prime}$ contribution is negligible.

If the maximum offset allowed across the FD is $X_{M}$, and:

$$
K_{o c t}=-\frac{4}{27} \frac{R_{11}^{3}}{X_{M}^{2} R_{12}} \text {, then } X_{F D}=X_{M} \cos \left(3 \arccos \left(\frac{X_{F D}^{o}}{3 X_{M}}\right)^{)}\right.
$$

The maximum for $X_{F D}^{o}$ is now $3 X_{M}$, so the depth of the horizontal collimators can be relaxed by a factor of 3 .

The octupoles can also make multiple tail foldings, using a method that could be defined as 'Chebyshev Arrangement of Octupoles'. For instance, if there are two octupoles with the following constraints:

$$
\begin{gathered}
K_{\text {oct_1 }}=-\frac{4}{243} \frac{T_{11} R_{11}^{2}}{X_{M}^{2} T_{12}} ; K_{\text {oct }_{-} 2}=-\frac{4}{27} \frac{R_{11}^{3}}{X_{M}^{2} S_{12} T_{11}^{3}} \\
S_{12}<<T_{12} R_{11}
\end{gathered}
$$

$R_{i j}, S_{i j}, T_{i j}$ are the first order transfer matrix elements between, respectively: the first octupole and the FD, the two octupoles, and the second octupole and the FD. Then:

$$
X_{F D}=X_{M}\left[\cos \left(9 \arccos \left(\frac{X_{F D}^{o}}{9 X_{M}}\right)\right)-\frac{4}{3} \frac{S_{12}}{T_{12} R_{11}}\left(\frac{X_{F D}^{o}}{9 X_{M}}\right)^{3}\right.
$$

produces about a factor of 9 'tail folding'. Figure 4 shows the changes in the phase space distribution due to two octupoles that meet these conditions.

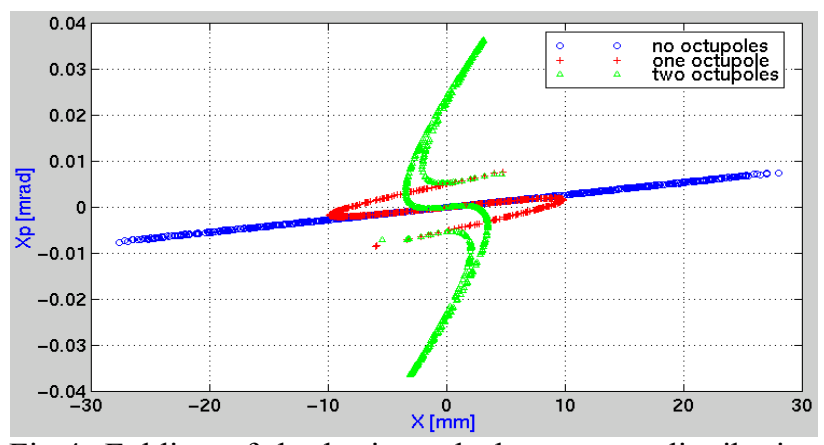

Fig.4: Folding of the horizontal phase space distribution at the entrance of the Final Doublet with one or two octupoles in a 'Chebyshev Arrangement'.

The situation is more complicated for the more general case of a particle with large offset in both planes. If an octupole focuses along the $\mathrm{x}$ and $\mathrm{y}$ axes, it will defocus the beam along the $45^{\circ}$ and $135^{\circ}$ axes. A system that has good 'folding' properties everywhere can quickly become very complicated. Large $\beta$ 's across the octupoles are also required, and the optics to produce these $\beta$ 's decreases the energy bandwidth. A simple solution is to use a pair of octupoles with opposite strengths, separated by a drift space, the net effect is roughly a fifth-order radial focusing. This can be easily seen with the following formalism. Assuming that a particle enters the doublet at 
$R=R e^{i \varphi}$ with no angle, it will exit the doublet with position and angle:

$$
\begin{aligned}
& R_{\text {out }}=R\left(1-K_{\text {oct }} L R^{2} e^{-4 i \varphi}\right) \\
& \theta_{\text {out }}=-3 K_{\text {oct }}^{2} L R^{4} R\left(1-K_{\text {oct }} L R^{2} e^{4 i \varphi}+\frac{1}{3}\left(K_{o c t} L R^{2} e^{4 i \varphi}\right)^{2}\right)
\end{aligned}
$$

$L$ is the drift between the 'thin' octupoles.

The focusing will be almost purely radial for all particles if $R<<\left(K_{\text {oct }} L\right)^{1 / 2}$.

A disadvantage of the Octupoles Doublets (OD) is that the octupoles have to be stronger in order to produce a significant folding. In addition, since now the folding is now fifth order, the doublet can only improve the stayclear by a factor 2 . There are several advantages: a) The ODs can be used in series. Two Chebyshev arranged OD's will provide a factor 4 tail folding. b) The ODs will not introduce any aberrations up to fourth order, so they do not affect the luminosity of the FF. c) The optics can very simple, as shown in Fig.1. Two ODs are placed in a drift space just downstream of the $\beta \mathrm{MS}$, with $\beta$-functions across them to have the optimal folding across both quadrupoles in the FD. d) With small readjustments of the relative strengths of the OD, the residual higher order non-radial focusing contributions can be further reduced.

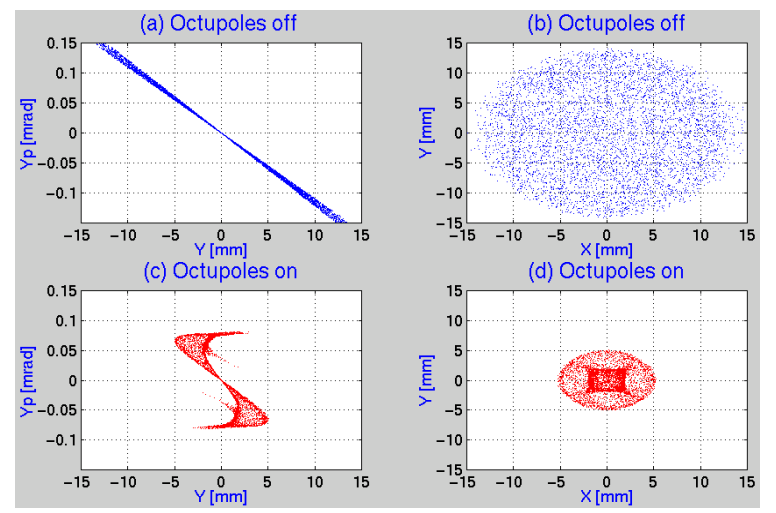

Fig.5: Effect of the ODs on the vertical phase-space and beam size at the first high $\beta_{y}$ point downstream. Input beam distributions are as in Fig.2.

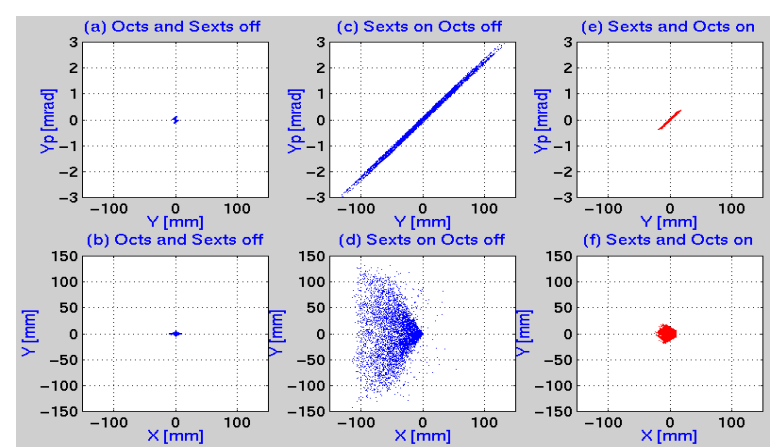

Fig.6: Effect of the ODs on the vertical phase space and beam size at the minimum $\beta$ point just upstream of the FD. Input beam distributions are as in Fig.2.

Figure $2 \mathrm{~d}$ shows that the ODs reduce the beam size across the FD by about a factor of four in both planes, limiting all the particles and the synchrotron radiation swath to within a circle of less then $10 \mathrm{~mm}$ radius. The off energy halo distribution is shown in Fig.3c/3f. Figure 5 shows the effect at the first high $\beta_{y}$ point downstream. Another advantage (Fig.6), is that the ODs also reduce the beam halo in the regions between the sextupoles, where the non-linear effects in the lattice are the strongest.

A preliminary study of the OD tolerances shows that, to avoid any loss of luminosity, about $10^{-3}$ field stability and about $5 \mu \mathrm{m}$ position stability is required

\section{INSERTIONS OF SCRAPERS}

The NLC collimation system was based on the original ZDR FF design [2], where the chromatic compensation was performed in dedicated sections. Such a system has more demanding collimation requirements because of the beam distributions shown in Fig.3g,h. In addition to the dedicated collimation section, additional scrapers had to be inserted into the FF itself due to the halo dilution in the FF. In the new FF with ODs, the total number of halo particles to be removed is extremely small. Under any normal running conditions, only a fraction of the particles produced by residual gas scattering in the Linac (about $10^{4}$ electrons per pulse [3]) should be outside the collimation limits of the FF. Even if the beam energy drops by $2 \%$ or more, there should be no background and the collider can continue to operate. The NLC collimation system can be redesigned to take advantage of this. The proposed integrated design of the collimation section and Final Focus is shown in Fig.1. The 'clean-up' collimators in the Final Focus can remove up to $10^{6}$ electrons per pulse, about $10^{-6}$ of the full beam charge, with an acceptable muon rate across the IP. In theory, the FF could provide all of the required collimation and the upstream system would only be needed for protection from errant pulses in the Linac.

\section{CONCLUSIONS}

The NLC FF design has been enhanced by the addition of a few non-linear elements, and other modifications. This system has better bandwidth, aperture and tolerances than the previous design. The engineering is much simpler, because there are fewer elements and more relaxed parameters. In addition, it could potentially eliminate much of the long, complex collimation system. The proposed Beam Delivery System is under $1.4 \mathrm{~km}$ long and can accommodate beams up to $5 \mathrm{TeV}$ in the center of mass.

\section{BIBLIOGRAPHY}

[1] P.Raimondi, A.Seryi, PRL 86, 3779 (2001).

[2] P.Tenenbaum, LCC note 0052 (2001).

[3] ZDR Report for the NLC, SLAC Report-474.

[4] P.F.Meads IEEE Trans Nuc Sci NS30, 1983, p.2838.

[5] N.Tsoupas, et al., PAC91, (1991), p.1695.

[6] F. Zimmermann, NLC Acc Phys Note, July 141998.

[7] R. Pitthan, SLAC-PUB-8402, 1999.

[8] R.Helm, SLAC, unpublished, 1999-2000.

[9] R.Brinkmann, BDIR2000 workshop, July 2000. 